NBI-HE-94-51, OU-HET 203

November 1994

hep-th/9411156

\title{
NOTE ON $W_{3}$ REALIZATIONS OF THE BOSONIC STRING
}

\author{
Fiorenzo Bastianelli円 \\ The Niels Bohr Institute, Blegdamsvej 17, DK-2100 Copenhagen Ø, Denmark \\ Nobuyoshi Ohta' \\ Department of Physics, Osaka University, Toyonaka, Osaka 560, Japan
}

\begin{abstract}
In order to investigate to what extent string theories are different vacua of a general string theory (the "universal string"), we discuss realizations of the bosonic string as particular background of certain types of $W$-strings. Our discussions include linearized $W_{3}^{l i n}$, non-critical $W_{3}$, linearized $W_{3}^{(2) l i n}$ and critical $W_{3}^{(2)}$ realizations of the bosonic string.
\end{abstract}

${ }^{1}$ e-mail address: fiorenzo@nbivax.nbi.dk

${ }^{2}$ e-mail address: ohta@phys.wani.osaka-u.ac.jp 


\section{Introduction}

Along the line of realization of various string theories in those with higher worldsheet gauge symmetries [1], those based on nonlinear algebras seem to belong to a very special class. The nonlinear character of these algebras makes it difficult to construct embeddings, and up to now only few special realizations have been discovered for the case of the bosonic string embedded in the $W$-strings [2, 3], 印. It would be quite interesting to examine further such realizations with the aim of finding general methods to describe embeddings. Our motivations arise from investigating to what extent string theories can be viewed as different vacua of a general string theory (the "universal string"). In this note we will present a few remarks and a few additional realizations of the bosonic string as particular background of certain types of $W$-strings. Our main idea is to use linearized versions of the $W$-algebras to find embeddings and to map these embeddings back to the nonlinear basis of the algebra. We could find embeddings for the linearized version of the $W$-algebras and prove their equivalence to the bosonic string, but we encountered technical difficulties in proving the equivalence in the nonlinear cases.

\section{$2 W_{3}^{\text {lin }}$ realization of the bosonic string}

An interesting approach to realize embeddings of the various string theories into those based on nonlinear algebras is to consider the linearization of these nonlinear algebras by including additional symmetry generators and performing nonlinear redefinitions of the generators. Recently Krivonos and Sorin [5] have constructed a linearized version of the $W_{3}$ algebra, which is denoted by $W_{3}^{\text {lin }}$ and is given by

$$
\begin{aligned}
T(z) T(w) & \sim \frac{c}{2} \frac{1}{(z-w)^{4}}+\frac{2 T(w)}{(z-w)^{2}}+\frac{\partial T(w)}{(z-w)} \\
T(z) G(w) & \sim \frac{x_{1} G(w)}{(z-w)^{2}}+\frac{\partial G(w)}{(z-w)} \\
T(z) J(w) & \sim \frac{J(w)}{(z-w)^{2}}+\frac{\partial J(w)}{(z-w)} \\
J(z) J(w) & \sim \frac{x_{2}}{(z-w)^{2}} \\
J(z) G(w) & \sim \frac{G(w)}{(z-w)}
\end{aligned}
$$

where the central charge $c$ and the coefficients $x_{1}, x_{2}$ are parametrized by

$$
c=\frac{1-4 x-9 x^{2}}{1+x}, \quad x_{1}=\frac{3}{2}+\frac{1}{1+x}, \quad x_{2}=1+x .
$$

An invertible nonlinear transformation maps this algebra into the usual nonlinear $W_{3}$ algebra extended by a spin 1 current. Such an extended $W_{3}$ algebra, which we will call $W_{3,1}$, is unique if one requires to preserve the usual $W_{3}$ algebra as subalgebra. This was discussed in ref. [5], to which we refer for the 
complete OPE of the $W_{3,1}$ algebra. In this section we will show how the bosonic string can be realized as a special case of the more general string that can be constructed by gauging the $W_{3}^{\text {lin }}$ algebra.

First we construct the BRST charge for the $W_{3}^{\text {lin }}$ algebra. It is given by

$$
Q=\oint\left[c_{t} T+c_{g} G+c_{j} J+c_{t}\left(\partial c_{t} b_{t}+\partial b_{g} c_{g}-b_{j} \partial c_{j}\right)+x_{1} \partial c_{t} b_{g} c_{g}+c_{j} b_{g} c_{g}\right]
$$

with the ghost correlators given by

$$
c_{\alpha}(z) b_{\beta}(w) \sim \frac{\delta_{\alpha \beta}}{(z-w)}, \quad \alpha, \beta=t, g, j .
$$

It is nilpotent for $x=-2$. This value implies $c=27, x_{1}=\frac{1}{2}, x_{2}=-1$. Note that the current $G$ acquires spin $\frac{1}{2}$ at the critical value $x=-2$, but it still has even Grassman parity.

Now we show that the bosonic string can be embedded into the more general string based on the $W_{3}^{l i n}$ algebra. Consider a consistent bosonic string background described by a conformal field theory with stress tensor $T_{m}$ satisfying the Virasoro algebra with central charge $c=26$. Tensoring it with a set of two commuting bc systems, $\left(\beta_{g}, \gamma_{g}\right),\left(\beta_{j}, \gamma_{j}\right)$ of spin $\left(\frac{1}{2}, \frac{1}{2}\right),(1,0)$, respectively, one can construct the following realization of the critical $W_{3}^{\text {lin }}$ algebra

$$
\begin{aligned}
T & =T_{m}+T\left(\beta_{g}, \gamma_{g}\right)+T\left(\beta_{j}, \gamma_{j}\right), \\
G & =\beta_{g}, \\
J & =\beta_{j}+\beta_{g} \gamma_{g},
\end{aligned}
$$

where we denote by $T(b, c)$ the stress tensor of a $(b, c)$ system of spin $(\lambda, 1-\lambda)$

$$
T(b, c)=-\lambda b \partial c+(1-\lambda) \partial b c
$$

Plugging the particular realization (5) into eq. (3), we get the following nilpotent BRST charge

$$
\begin{aligned}
\tilde{Q}= & \oint\left[c_{t}\left(T_{m}+\frac{1}{2} T\left(b_{t}, c_{t}\right)+T\left(b_{g}, c_{g}\right)+T\left(b_{j}, c_{j}\right)+T\left(\beta_{g}, \gamma_{g}\right)+T\left(\beta_{j}, \gamma_{j}\right)\right)\right. \\
& \left.+c_{g} \beta_{g}+c_{j}\left(\beta_{j}+\beta_{g} \gamma_{g}+b_{g} c_{g}\right)\right] .
\end{aligned}
$$

It is canonically equivalent to the BRST charge of the bosonic string plus a sector of non-minimal terms which decouples and has trivial cohomology. In fact one can check that

$$
\mathrm{e}^{R_{2}} \mathrm{e}^{R_{1}} \tilde{Q} \mathrm{e}^{-R_{1}} \mathrm{e}^{-R_{2}}=Q_{\text {bos }}+Q_{n m},
$$

where $Q_{b o s}$ is the BRST of the usual bosonic string

$$
Q_{b o s}=\oint c_{t}\left(T_{m}+\frac{1}{2} T\left(b_{t}, c_{t}\right)\right),
$$


and where

$$
\begin{aligned}
Q_{n m} & =\oint\left(c_{g} \beta_{g}+c_{j} \beta_{j}\right), \\
R_{1} & =-\oint c_{j} b_{g} \gamma_{g}, \\
R_{2} & =-\oint c_{t}\left(T\left(b_{g}, \gamma_{g}\right)+T\left(b_{j}, \gamma_{j}\right)\right) .
\end{aligned}
$$

The topological charge $Q_{n m}$ imposes the constraint that the fields $\left(\beta_{g}, \gamma_{g}, b_{g}, c_{g}\right)$ as well as $\left(\beta_{j}, \gamma_{j}, b_{j}, c_{j}\right)$ make quartets and decouple from the theory. Thus the bosonic string is realized as a particular background of the $W_{3}^{\text {lin }}$-string.

\section{$3 \quad$ Finding quadratic $W_{3}$}

The realization (5) can be used to find a realization of nonlinear $W_{3}$ as follows. Define the generators

$$
\begin{aligned}
T_{W} & =T-\frac{5}{2} \partial J \\
& =T_{m}-3 \beta_{g} \partial \gamma_{g}-2 \partial \beta_{g} \gamma_{g}-\beta_{j} \partial \gamma_{j}-\frac{5}{2} \partial \beta_{j}, \\
W & =G+2 i \sqrt{\frac{2}{133}}\left[J T+\frac{2}{3} J^{3}+\frac{5}{2} J \partial J+\frac{5}{4} \partial T+\frac{25}{24} \partial^{2} J\right] \\
& =\beta_{g}+i \sqrt{\frac{2}{133}}\left(2 \beta_{g} \gamma_{g} T_{m}+2 \beta_{j} T_{m}+\frac{4}{3} \beta_{j}^{3}+4 \beta_{g} \gamma_{g} \beta_{j}^{2}+5 \beta_{g} \gamma_{g} \partial \beta_{j}+4 \beta_{g}^{2} \gamma_{g}^{2} \beta_{j}\right. \\
& +\frac{4}{3} \beta_{g}^{3} \gamma_{g}^{3}+10 \beta_{g} \partial \beta_{g} \gamma_{g}^{2}+10 \partial \beta_{g} \gamma_{g} \beta_{j}-2 \beta_{g} \gamma_{g} \beta_{j} \partial \gamma_{j}+7 \partial^{2} \beta_{g} \gamma_{g}-\frac{1}{2} \partial \beta_{g} \partial \gamma_{g} \\
& \left.-\frac{1}{2} \beta_{g} \partial^{2} \gamma_{g}+5 \beta_{j} \partial \beta_{j}-2 \beta_{j}^{2} \partial \gamma_{j}-\frac{5}{2} \partial \beta_{j} \partial \gamma_{j}-\frac{5}{2} \beta_{j} \partial^{2} \gamma_{j}+\frac{5}{2} \partial T_{m}+\frac{25}{12} \partial^{2} \beta_{j}\right),
\end{aligned}
$$

where $T, G, J$ are given in (5). One may verify that the generators $\left(T_{W}, W, J\right)$ realize a representation of the $W_{3,1}$ algebra with the correct amount of central charge $(c=102)$ expected to balance the ghost contribution. Thus one may conjecture that it defines a critical realization of the $W_{3,1}$ algebra. However to prove such a conjecture one has to construct the BRST charge for $W_{3,1}$ and verify that the above realization makes it nilpotent. The construction of such a BRST charge is tedious because the algebra closes with cubic relations and it is not discussed here.

An intriguing alternative is to notice that $\left(T_{W}, W\right)$ in (11) satisfy the $W_{3}$ OPE with central charge $c=102$. This realization is interesting in that the generator $W$ contains a linear term $\beta_{g}$, indicating the spontaneous breakdown of the symmetry. This is quite different from the realizations discussed in refs. [2, 团, in which there is a linear term but with derivatives. However the algebra satisfied by these generators has the non-critical central charge $c=102$ and we have the "extra" matter $\beta_{j}, \gamma_{j}$. One can still define a nilpotent BRST operator by coupling the $W_{3}$ matter system described by $\left(T_{W}, W\right)$ to a "Liouville" system à la ref. [6]. For this purpose, we need a $W_{3}$ realization with central charge $c=-2$ 
as a "Liouville" system. We find that

$$
\begin{aligned}
T_{L} & =-\eta_{L} \partial \xi_{L}, \\
W_{L} & =\frac{1}{\sqrt{6}}\left(\partial \eta_{L} \partial \xi_{L}-\eta_{L} \partial^{2} \xi_{L}\right),
\end{aligned}
$$

satisfy the $W_{3}$ algebra with $c=-2$, where $\left(\eta_{L}, \xi_{L}\right)$ are anticommuting fields. Using this realization, the nilpotent BRST operator is given by

$$
\begin{aligned}
Q= & \oint\left[c_{t}\left(T_{W}+T_{L}\right)+i \frac{\sqrt{133}}{2} c_{w} W+\frac{\sqrt{3}}{2} c_{w} W_{L}+\left(T_{L}-T_{W}\right) b_{t} c_{w} \partial c_{w}\right. \\
& \left.+c_{t} \partial c_{t} b_{t}-3 c_{t} b_{w} \partial c_{w}-2 c_{t} \partial b_{w} c_{w}-\frac{13}{8} \partial b_{t} c_{w} \partial^{2} c_{w}-\frac{65}{24} b_{t} c_{w} \partial^{3} c_{w}\right] .
\end{aligned}
$$

Since we have the linear term $\beta_{g}$ in $W$ which can be used to decouple $\left(\beta_{g}, \gamma_{g}, b_{w}, c_{w}\right)$ as in the superstrings [1], we expect that this model contains the bosonic string as a subsector without states from these fields. Unfortunately a constraint that would eliminate the fields $\left(\eta_{L}, \xi_{L}, \beta_{j}, \gamma_{j}\right)$ does not seem to be present in the BRST charge, so it may be that additional states are present, as in the model of ref. 狛] where a construction reminiscent of ours has been presented. It would be interesting to examine the full cohomology of eq. (13).

Before closing this section, we would like to note that eq. (11) suggests that it may be possible to find a critical realization using only $\left(\beta_{g}, \gamma_{g}\right)$ with a linear $\beta_{g}$ term in $W$, unlike that of refs. 22, 4. However terms like $\partial^{2} \gamma_{g}$ having zero dimension can appear with an arbitrary power, making it harder to proceed systematically in the construction of the generators. For example, a possible method to find such a realization is to use an expansion in ghost number as in ref. [7]. Namely we assign ghost number -1 to $\beta_{g}$ and 1 to $\gamma_{g}$, and assume the following form

$$
\begin{aligned}
T & =T_{m}-3 \beta_{g} \partial \gamma_{g}-2 \partial \beta_{g} \gamma_{g}+(\text { possible terms with ghost number } 2)+\cdots, \\
W & =\beta_{g}+(\text { possible terms with ghost number } 1)+\cdots
\end{aligned}
$$

Then one can try to satisfy the OPE for $W_{3}$ at each ghost number. Unfortunately the calculation involves increasingly large number of terms for increasing ghost numbers and it seems difficult to construct a realization in this way.

\section{$4 W_{3}^{(2) l i n}$ realization of the bosonic string}

In ref. [5] another nonlinear algebra $W_{3}^{(2)}$ [8] was also linearized. The linearized version, called $W_{3}^{(2) l i n}$, is given by

$$
T(z) T(w) \sim \frac{(7-9 x) x}{2(x+1)} \frac{1}{(z-w)^{4}}+\frac{2 T(w)}{(z-w)^{2}}+\frac{\partial T(w)}{(z-w)},
$$




$$
\begin{aligned}
T(z) J(w) & \sim \frac{J(w)}{(z-w)^{2}}+\frac{\partial J(w)}{(z-w)}, \\
T(z) G^{ \pm}(w) & \sim \frac{3}{2} \frac{G^{ \pm}(w)}{(z-w)^{2}}+\frac{\partial G^{ \pm}(w)}{(z-w)}, \\
J(z) J(w) & \sim \frac{x}{(z-w)^{2}}, \quad J(z) G^{ \pm}(w) \sim \pm \frac{G^{ \pm}(w)}{(z-w)}, \\
T(z) K(w) & \sim-\frac{1}{2} \frac{K(w)}{(z-w)^{2}}+\frac{\partial K(w)}{(z-w)}, \quad J(z) K(w) \sim \frac{K(w)}{(z-w)}, \\
G^{-}(z) K(w) & \sim \frac{1}{(z-w)} .
\end{aligned}
$$

The standard procedure for constructing the BRST operator fails to give a nilpotent BRST charge. This is presumably related to the fact that not all possible central charges compatible with the Jacobi identities are present in the OPE (15). In fact one can write down all possible central charges $\left(c, c_{1}, c_{2}, c_{3}, x, x_{1}\right)$ that can appear by dimensional analysis

$$
\begin{aligned}
T(z) T(w) & \sim \frac{c}{2} \frac{1}{(z-w)^{4}}+\frac{2 T(w)}{(z-w)^{2}}+\frac{\partial T(w)}{(z-w)}, \\
T(z) J(w) & \sim \frac{c_{1}}{(z-w)^{3}}+\frac{J(w)}{(z-w)^{2}}+\frac{\partial J(w)}{(z-w)}, \\
T(z) G^{ \pm}(w) & \sim \frac{3}{2} \frac{G^{ \pm}(w)}{(z-w)^{2}}+\frac{\partial G^{ \pm}(w)}{(z-w)}, \\
J(z) J(w) & \sim \frac{x}{(z-w)^{2}}, \quad J(z) G^{ \pm}(w) \sim \pm \frac{G^{ \pm}(w)}{(z-w)}, \\
T(z) K(w) & \sim-\frac{1}{2} \frac{K(w)}{(z-w)^{2}}+\frac{\partial K(w)}{(z-w)}, \quad J(z) K(w) \sim \frac{K(w)}{(z-w)}, \\
G^{-}(z) K(w) & \sim \frac{x_{1}}{(z-w)}, \\
G^{+}(z) G^{-}(w) & \sim \frac{c_{2}}{(z-w)^{3}} .
\end{aligned}
$$

Then the standard BRST charge

$$
\begin{aligned}
Q= & \oint\left[c_{t} T+c_{j} J+c_{+} G^{+}+c_{-} G^{-}+c_{k} K+c_{t} \partial c_{t} b_{t}+c_{t} \partial b^{+} c_{+}+\frac{3}{2} \partial c_{t} b^{+} c_{+}+c_{t} \partial b^{-} c_{-}\right. \\
& \left.+\frac{3}{2} \partial c_{t} b^{-} c_{-}-c_{t} b_{j} \partial c_{j}+c_{j}\left(b^{+} c_{+}-b^{-} c_{-}\right)+c_{t} \partial b_{k} c_{k}-\frac{1}{2} \partial c_{t} b_{k} c_{k}+c_{j} b_{k} c_{k}\right],
\end{aligned}
$$

is nilpotent for $c=61, c_{1}=2, x=-3$ and $x_{1}=c_{2}=c_{3}=0$. In general these central charges are not all independent and should be fixed by the Jacobi identities. Since we are only interested in the critical algebra, we have not worked out all the relations arising from Jacobi identities. It is enough to know that the nilpotency of the BRST charge guarantees that the Jacobi identities are satisfied for the critical algebra, for which we will indeed find an explicit realization.

The bosonic string can be embedded in the string described by the algebra (16) by introducing, along with a consistent bosonic string background given by the stress tensor $T_{m}$, four commuting $b c$ systems 
$\left(\beta^{+}, \gamma_{+}\right),\left(\beta^{-}, \gamma_{-}\right),\left(\beta_{k}, \gamma_{k}\right),\left(\beta_{j}, \gamma_{j}\right)$ of spin $\left(\frac{3}{2},-\frac{1}{2}\right),\left(\frac{3}{2},-\frac{1}{2}\right)\left(-\frac{1}{2}, \frac{3}{2}\right),(1,0)$, respectively. The generators

$$
\begin{aligned}
T & =T_{m}+T\left(\beta^{+}, \gamma_{+}\right)+T\left(\beta^{-}, \gamma_{-}\right)+T\left(\beta_{k}, \gamma_{k}\right)+T\left(\beta_{j}, \gamma_{j}\right), \\
G^{+} & =\beta^{+}, \quad G^{-}=\beta^{-}, \quad K=\beta_{k}, \\
J & =\beta_{j}+\beta^{+} \gamma_{+}-\beta^{-} \gamma_{-}+\beta_{k} \gamma_{k},
\end{aligned}
$$

realize the critical algebra (16). Plugging this realization in eq. (17), we obtain a BRST charge $\tilde{Q}$ that is canonically equivalent to the BRST charge of the bosonic string $Q_{b o s}$ plus a sector of non-minimal terms which decouples and carries trivial cohomology. In fact one can check that

$$
\mathrm{e}^{R_{2}} \mathrm{e}^{R_{1}} \tilde{Q} \mathrm{e}^{-R_{1}} \mathrm{e}^{-R_{2}}=Q_{\text {bos }}+Q_{n m}
$$

where

$$
\begin{aligned}
Q_{n m} & =\oint\left(c_{+} \beta^{+}+c_{-} \beta^{-}+c_{k} \beta_{k}+c_{j} \beta_{j}\right) \\
R_{1} & =-\oint c_{j}\left(b^{+} \gamma_{+}-b^{-} \gamma_{-}+b_{k} \gamma_{k}\right) \\
R_{2} & =-\oint c_{t}\left(T\left(b^{+}, \gamma_{+}\right)+T\left(b^{-}, \gamma_{-}\right)+T\left(b_{k}, \gamma_{k}\right)+T\left(b_{j}, \gamma_{j}\right)\right)
\end{aligned}
$$

One problem with this construction is that the nilpotency of the BRST charge (17) requires $x_{1}=0$, but this must be non-vanishing in order to reproduce the $W_{3}^{(2)}$ algebra as a subalgebra of (16). Another approach that allows to construct a nilpotent operator from the algebra (15) and avoids this problem is that of introducing an additional commuting $(\beta, \gamma)$ system of $\operatorname{spin}\left(\frac{3}{2},-\frac{1}{2}\right)$ so that

$$
\begin{aligned}
Q= & \oint\left[c_{t} T+c_{j} J+c_{+} G^{+}+c_{-} G^{-}+c_{k} K+c_{t} \partial c_{t} b_{t}+c_{t} \partial b^{+} c_{+}+\frac{3}{2} \partial c_{t} b^{+} c_{+}+c_{t} \partial b^{-} c_{-}\right. \\
& +\frac{3}{2} \partial c_{t} b^{-} c_{-}-c_{t} b_{j} \partial c_{j}+c_{j}\left(b^{+} c_{+}-b^{-} c_{-}\right)+c_{t} \partial b_{k} c_{k}-\frac{1}{2} \partial c_{t} b_{k} c_{k}+c_{j} b_{k} c_{k} \\
& \left.-\frac{3}{2} c_{t} \beta \partial \gamma-\frac{1}{2} c_{t} \partial \beta \gamma-c_{j} \beta \gamma+c_{-} \beta+c_{k} \gamma\right],
\end{aligned}
$$

is nilpotent for $x=-2$ or central charge $c=50$. Note that the first two lines of eq. (21) give the charge naively expected which however is not nilpotent. Actually this approach is just a reinterpretation of the previous one. We can read off the total matter generators by taking anticommutators of (21) with the respective ghosts and dropping the ghost part. We find

$$
\begin{aligned}
T^{\prime} & =T+T(\beta, \gamma), \quad J^{\prime}=J-\beta \gamma, \\
G^{+^{\prime}} & =G^{+}, \quad G^{-^{\prime}}=G^{-}+\beta, \quad K^{\prime}=K+\gamma .
\end{aligned}
$$

We see that these are modified by $\beta, \gamma$ such that they satisfy the critical algebra (16). This suggests that the generators $G^{-}$and $K$ should be spontaneously broken because the total generators $G^{-^{\prime}}$ and $K^{\prime}$ 
contain linear terms. It is also possible to eliminate $c_{-}, b^{-}, \beta, \gamma$, at the cost of redefining the generators. The BRST charge (21) can be transformed as

$$
\begin{aligned}
e^{R} Q e^{-R}= & \oint\left[c_{t} T+c_{j} J+c_{+} G^{+}+c_{t} \partial c_{t} b_{t}+c_{t} \partial b^{+} c_{+}+\frac{3}{2} \partial c_{t} b^{+} c_{+}-c_{t} b_{j} \partial c_{j}\right. \\
& \left.+c_{t} \partial G^{-} K+\frac{3}{2} \partial c_{t} G^{-} K+c_{j}\left(b^{+} c_{+}-G^{-} K\right)+c_{k} K+c_{-} \beta\right]
\end{aligned}
$$

where

$$
R=\oint\left[-\gamma G^{-}-c_{t}\left(T\left(b^{-}, \gamma\right)+T\left(b_{k}, G^{-}\right)\right)+c_{j}\left(b^{-} \gamma-b_{k} G^{-}\right)\right] .
$$

The last term in eq. (23) implies that the four fields $\left(b^{-}, c_{-}, \beta, \gamma\right)$ make a quartet and decouple from the theory. The generators corresponding to $G^{-}, K$ obtained from (23) by the anticommutators with $b^{-}, b_{k}$ decouple from the rest of the generators. This is an implementation through similarity transformations of the procedure of eliminating $G^{-}, K$ by redefinitions given in ref. [5].

We can find a realization of the algebra (15) for $x=-2$, which is given by

$$
\begin{aligned}
T & =T_{m}+T\left(\beta^{+}, \gamma_{+}\right)+T\left(\beta^{-}, \gamma_{-}\right)+T\left(\beta_{j}, \gamma_{j}\right), \\
G^{+} & =\beta^{+}, \quad G^{-}=\beta^{-}, \quad K=-\gamma_{-}, \\
J & =\beta_{j}+\beta^{+} \gamma_{+}-\beta^{-} \gamma_{-} .
\end{aligned}
$$

Now we can show the equivalence to bosonic string. The BRST charge (21) with eq. (25) substituted in can be transformed as

$$
e^{R_{2}} e^{R_{1}} Q e^{-R_{1}} e^{-R_{2}}=Q_{b o s}+Q_{n m},
$$

where $Q_{b o s}$ is that for the bosonic string given in eq. (9) and $Q_{n m}$ is that for the non-minimal sector

$$
Q_{n m}=\oint\left[c_{j} \beta_{j}+c_{+} \beta^{+}+c_{-}\left(\beta^{-}+\beta\right)-c_{k}\left(\gamma_{-}-\gamma\right)\right],
$$

and where

$$
\begin{aligned}
R_{1}= & \oint\left[\frac{1}{2} \gamma_{j}\left(-\beta^{+} \gamma_{+}+\beta^{-} \gamma_{-}+\beta \gamma-b^{+} c_{+}+b^{-} c_{-}-b_{k} c_{k}\right)\right. \\
& \left.+\frac{1}{4} c_{j}\left(-2 b^{+} \gamma_{+}+b^{-}\left(\gamma_{-}+\gamma\right)-b_{k}\left(\beta^{-}-\beta\right)\right)\right] \\
R_{2}= & -\oint c_{t}\left[T\left(b^{+}, \gamma_{+}\right)+T\left(b^{-}, \frac{\gamma_{-}+\gamma}{2}\right)+T\left(b_{k}, \frac{\beta^{-}-\beta}{2}\right)+T\left(b_{j}, \gamma_{j}\right)\right] .
\end{aligned}
$$

Again the non-minimal term imposes the condition that all fields except those in the original bosonic string decouple from the physical subspace and the theory is equivalent to the bosonic string.

\section{$5 \quad W_{3}^{(2)}$ realization of the bosonic string}

The unorthodox way of constructing a nilpotent BRST charge for the linear algebra in eq. (15), presented in the second part of the previous paragraph, gives us a hint on how to obtain a realization of 
the bosonic string as a particular background for the nonlinear $W_{3}^{(2)}$-string. To show this, we first note that the $W_{3}^{(2)}$ BRST charge [9] is given by

$$
\begin{aligned}
Q & =\oint\left[c_{t} T+c_{+} \hat{G}^{+}+c_{-} G^{-}+c_{j} J+c_{t}\left(\frac{1}{2} T\left(b_{t}, c_{t}\right)+T\left(b^{+}, c_{+}\right)+T\left(b^{-}, c_{-}\right)+T\left(b_{j}, c_{j}\right)\right)\right. \\
& \left.+b_{t} c_{+} c_{-}+\frac{3}{2} b_{j}\left(c_{+} \partial c_{-}-\partial c_{+} c_{-}\right)+c_{j}\left(b^{+} c_{+}-b^{-} c_{-}\right)-\frac{2}{x+1} b_{j} c_{+} c_{-} J\right],
\end{aligned}
$$

where the spin $\frac{3}{2}$ generator $\hat{G}^{+}$is given by the nonlinear redefinition of Krivonos and Sorin [5]

$$
\begin{aligned}
\hat{G}^{+} & =G^{+}+T K-\frac{2}{x+1} J^{2} K-\frac{3 x+7}{2(x+1)} \partial J K+\frac{2}{x+1} J G^{-} K^{2}-\frac{2}{3(x+1)} G^{-} G^{-} K^{3} \\
& -3 K \partial K G^{-}+\frac{1-x}{1+x} K^{2} \partial G^{-}+3 \partial(J K)-\frac{3(x+1)}{2} \partial^{2} K
\end{aligned}
$$

such that $\left(T, \hat{G}^{+}, G^{-}, J\right)$ constitute a $W_{3}^{(2)}$ algebra. We find that the nilpotency condition for this charge is precisely $x=-2$ or $c=50$. Hence substituting the critical realization given in eq. (25), we get an embedding of the bosonic string. The conjecture that this BRST charge is canonically equivalent to that of the bosonic string plus a sector of non-minimal fields may be proved by performing similarity transformations, but we have not been able to find the set of transformations that does the job.

\section{Conclusions}

We have presented a few realizations of the bosonic string as a background for some $W$-strings and discussed their properties. We have used linearized versions of the $W$-algebras to identify the embeddings. For the $W_{3}$ case we have obtained a non-critical realization. Adding to it a "Liouville" system to obtain criticality we have achieved a critical embedding. However the resulting theory presumably contains additional states on top of those present in the bosonic string. In fact a full equivalence could not be established. In the $W_{3}^{(2)}$ case the linearized embedding has helped us to identify a consistent background for the $W_{3}^{(2)}$-string that might reproduce the full structure of the bosonic string without additional states. Thus the $W_{3}^{(2)}$ case seems to be simpler than the $W_{3}$ case in that we could identify a critical embedding. However we have not found the correct similarity transformation that proves the complete equivalence, even though we suspect this should be possible. The $W_{3}$ and $W_{3}^{(2)}$ algebras are closely related. In fact $W$-algebras can generically be derived as hamiltonian reduction of WZNW models, and the specific case of the $W_{3}$ and $W_{3}^{(2)}$ algebras correspond to different embedding of $\operatorname{sl}(2)$ into $\operatorname{sl}(3)$ [10. Perhaps the method of hamiltonian reduction could be successfully used in the search for a "universal string".

\section{Acknowledgements}

We have checked many of our OPEs using the Mathematica package developed by K. Thielemans [11. We would like to thank H. Kunitomo and J.L. Petersen for valuable discussions. The work of F.B. was supported by the EU grant no. ERBCHBGCT930407. 


\section{References}

[1] N. Berkovits and C. Vafa, Mod. Phys. Lett. A9 (1994) 653;

J. M. Figueroa-O'Farrill, Phys. Lett. B321 (1994) 344;

H. Ishikawa and M. Kato, Mod. Phys. Lett. A9 (1994) 725;

F. Bastianelli, Phys. Lett. B322 (1994) 340;

N. Ohta and J. L. Petersen, Phys. Lett. B325 (1994) 67;

F. Bastianelli, N. Ohta and J. L. Petersen, Phys. Lett. B327(1994) 35; Phys. Rev. Lett. 73 (1994) 1199.

[2] N. Berkovits, M. Freeman and P. West, Phys. Lett. B325 (1994) 63.

[3] H. Kunitomo, M. Sakaguchi and A. Tokura, preprints OU-HET-187, hep-th/9403086, OU-HET-199, hep-th/9408007.

[4] E. Bergshoeff, H.J. Boonstra and M. de Roo, preprint UG-7/94, hep-th/9409186.

[5] S. O. Krivonos and A. S. Sorin, Phys. Lett. B335 (1994) 45.

[6] M. Bershadsky, W. Lerche, D. Nemeschansky and N. P. Warner, Phys. Lett. B292 (1992) 35.

[7] N. Berkovits and N. Ohta, Phys. Lett. B334 (1994) 72.

[8] A. Polyakov, Int. J. Mod. Phys. A5 (1990) 833;

M. Bershadsky, Comm. Math. Phys. 139 (1991) 71.

[9] Z. Khviengia and E. Sezgin, Phys. Lett. B326 (1994) 243.

[10] F.A. Bais, T. Tjin and P. van Driel, Nucl. Phys. B357 (1991) 632.

[11] K. Thielemans, Int. J. Mod. Phys. C3 (1991) 787. 